\title{
The functional role of protein dynamics in photosynthetic reaction centers investigated by elastic and quasielastic neutron scattering
}

\author{
Jörg Pieper ${ }^{\mathrm{a}}$ \\ Tartu University, Institute of Physics, Tartu, Estonia
}

\begin{abstract}
This short review summarizes our current knowledge about the functional relevance of protein dynamics in photosynthetic reaction centers. In the case of Photosystem II membrane fragments, elastic and quasielastic neutron scattering experiments reveal a dynamical transition at about $240 \mathrm{~K}$ corresponding to the activation of picosecond molecular motions. Likewise, a "freezing" of molecular dynamics is observed upon dehydration. Intriguingly, these effects correlate with the pronounced temperature- and hydration-dependence of specific electron transfer steps in Photosystem II indicating that molecular dynamics is an indispensable prerequisite for its function. Thus, electron transfer in Photosystem II appears to be a prototypical example for a dynamics-function correlation. Finally, the laser-neutron pump-probe technique is shown to permit in-situ monitoring of molecular dynamics in specific functional states of a protein in real time.
\end{abstract}

\section{Introduction}

Photosynthesis is a fundamental natural process that enables the conversion of solar energy into storable chemical energy under the release of molecular oxygen derived from water. The essential processes of photosynthetic water splitting [1,2] take place in a membrane-bound protein assembly denoted as Photosystem II (PS II). The structure of the PS II core at $3.8 \AA$ resolution [3] is shown in Fig. 1. The PS II core comprises two different types of pigment-protein complexes with distinct functional designation: i) the light-harvesting complexes $\mathrm{CP} 43$ and $\mathrm{CP} 47$ and ii) the reaction center $(\mathrm{RC})$ represented by the central heterodimer D1/D2, where a light-triggered charge separation initiates the photochemical processes in PS II $[1,2]$.

The arrangement of the involved cofactors is shown in Fig. 2 (see [1-3] and references therein). In brief, the primary charge separation leads to the generation of an ion radical pair $\mathrm{P} 60^{+} \mathrm{Pheo}^{-}$and subsequently to electron transfer from Pheo to a plastoquinone molecule $\mathrm{Q}_{\mathrm{A}}$ within about $300 \mathrm{ps}$ [4]. Further electron transfer from $\mathrm{Q}_{\mathrm{A}}$ to a transiently bound plastoquinone $\mathrm{Q}_{\mathrm{B}}$ along with coupled proton uptake reactions leads to plastoquinol formation [5]. Eventually, the presence of $\mathrm{P} 680^{+}$induces the oxidative water splitting at a manganese containing water oxidizing complex (see [1] and references therein).

Specific redox reactions in the PS II RC exhibit a strong dependence on temperature and hydration. For example, the light-induced primary charge separation remains fully functional even at very low temperatures, while the $\mathrm{Q}_{\mathrm{A}} \rightarrow \mathrm{Q}_{\mathrm{B}}$ electron transfer is completely inhibited below about $200 \mathrm{~K}$ [6] and below a hydration of $45 \%$ relative humidity (r.h.) [7]. Likewise, individual reaction steps of the water oxidizing complex possess

\footnotetext{
${ }^{a}$ Corresponding author: pieper@ut.ee
}

a characteristic dependence on temperature (see [1] and references therein). The latter effects may indicate that protein dynamics is an essential prerequisite for functional processes in PS II.

In this regard, elastic, quasi-, and inelastic neutron scattering (ENS, QENS, and INS, respectively) are valuable experimental tools for direct studies of diffusive molecular and vibrational dynamics in biomolecules (for reviews see [8,9]). So far, ENS and QENS have been used to study the temperature and hydration dependence of molecular dynamics in PS II membranes of green plants [10-14] as well as that of bacterial RCs $[15,16]$. This review provides a short overview about the results of these studies.

\section{QENS studies of PS II membranes}

QENS studies of PS II membrane fragments were initially carried out varying sample temperature [10] and hydration [11]. For this purpose, PS II membrane fragments were prepared from spinach thylakoids as described in detail in [10]. The samples were hydrated using $\mathrm{D}_{2} \mathrm{O}$ in order to suppress the hydration water scattering and equilibrated at well defined r.h.-values using vapors of saturated salt solutions. A scheme of the sample system is shown in the upper left corner of Fig. 3 depicting a hydrated pseudostack of PS II membrane fragments with a) RC, b) core and minor antenna complexes, and c) the major antenna complex LHC II.

The QENS spectra shown in Fig. 3 [11] for three representative hydration levels were obtained with an elastic resolution of $93 \mu \mathrm{eV}$ corresponding to a time resolution of about $20 \mathrm{ps}$. These data show that sizeable quasielastic contributions are visible only for hydration levels higher than $44 \%$ r.h., while their relative intensities are increasing with increasing hydration. The spectra were fit using a Lorentzian component with a width

This is an Open Access article distributed under the terms of the Creative Commons Attribution License 4.0, which permits unrestricted use, distribution, and reproduction in any medium, provided the original work is properly cited. 


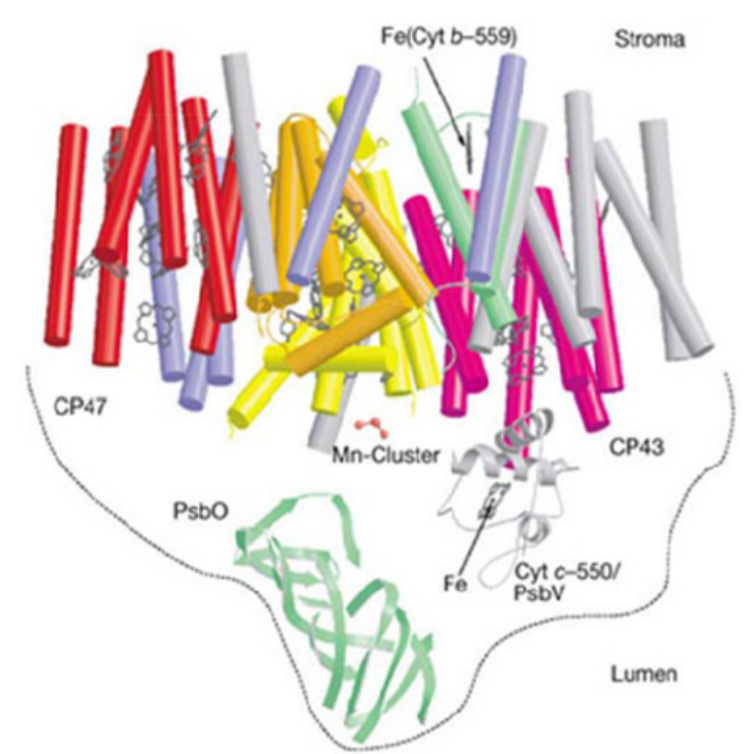

Figure 1. Structure of PSII of Synechococcus elongatus according to Zouni et al. [3] viewed along the membrane normal. The transmembrane $\alpha$-helices of the central D1/D2 heterodimer are shown as yellow and orange cylinders, respectively. The core antenna complexes CP43 and CP47 are indicated in purple and red, respectively. The PsbO protein is shown as a green $\beta$-sheet structure and Cytochrome c-550 as a helical model. The cofactor arrangement is shown in more detail in Fig. 2. This figure is reprinted from Zouni et al. [3] with permission.

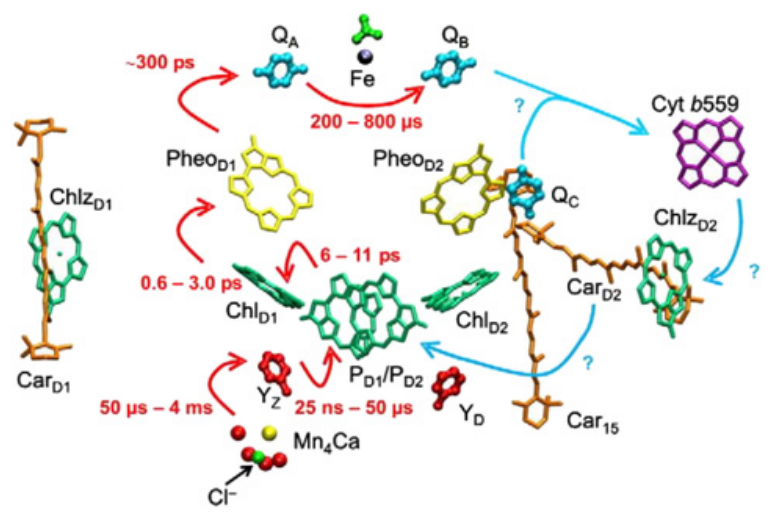

Figure 2. Arrangement of the cofactors within the PS II RC seen along the membrane normal. Chlorophylls (Chl) are coloured in green, pheophytins (Pheo) in yellow, plastoquinones in light blue and carotenoids in brown, respectively. The red arrows indicate the directions of light-induced charge separation, water splitting, and plastoquinol formation. This figure is reprinted from [2] with permission.

(HWHM) of $0.1135 \mathrm{meV}$ corresponding to a correlation time of $5.8 \mathrm{ps}$. This result illustrates that hydration strongly affects diffusive molecular dynamics in PS II so that the hydration water plays the role of a "lubricant" for the molecular flexibility. Similar hydration effects on dynamics were reported before for other biomolecular systems including the purple membrane of Halobacterium salinarum $[17,18]$.

QENS experiments were also employed to study the temperature dependence of the molecular dynamics of

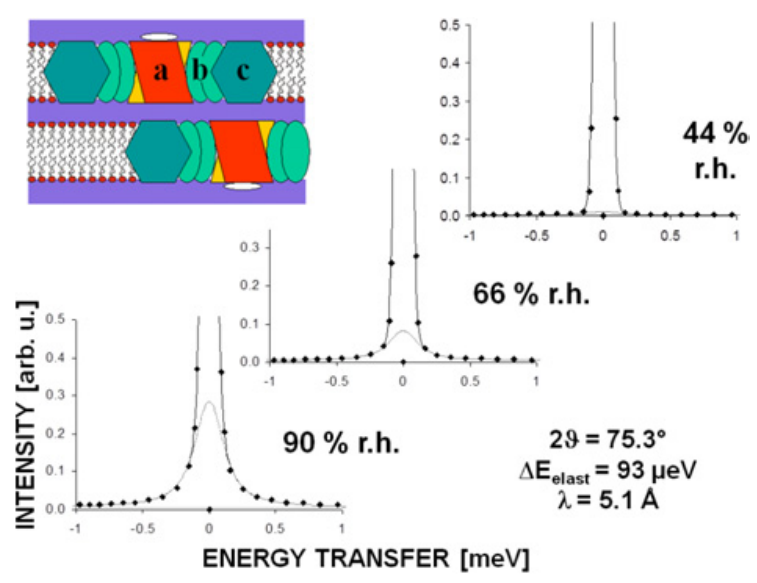

Figure 3. QENS spectra (black diamonds) of PS II membrane fragments hydrated with $\mathrm{D}_{2} \mathrm{O}$ at 44,66 , and $90 \%$ r.h., respectively, obtained at the time-of-flight spectrometer NEAT with an incident neutron wavelength of $5.1 \AA$ and an elastic resolution of $93 \mu \mathrm{eV}$ (about $20 \mathrm{ps}$ ) at a scattering angle of $75.3^{\circ}$ at $300 \mathrm{~K}$ according to [11]. Black full lines show normalized Lorentzian fits. The elastic peak is cut off at a value of 0.5 for ease of inspection. The inset shows a schematic view of the hydrated PS II membrane sample with a) reaction center, b) core and minor antennae and c) major antenna complexes. The hydration water is indicated in purple. This figure is reprinted from [11] with permission.

PS II membrane fragments hydrated at $90 \%$ r.h. [10]. The QENS spectra could be fit with two Lorentzian components corresponding to relaxation times of about $19 \mathrm{ps}$ and $0.8 \mathrm{ps}$, respectively, at all temperatures. The temperature dependence of the corresponding QISFs, hereafter denoted as $\mathrm{QISF}_{1}$ and $\mathrm{QISF}_{2}$ for the $19 \mathrm{ps}$ and $0.8 \mathrm{ps}$ components, respectively, are shown in Fig. 4. Three temperature ranges can be distinguished: a) at temperatures lower than $\sim 120 \mathrm{~K}$ (range $\mathrm{A}$ ), there is virtually no quasielastic contribution indicating that conformational molecular motions are frozen, b) at temperatures above $120 \mathrm{~K}$ (range B), molecular motions corresponding to QISF $_{1}$ are present, which are often identified with those of methyl groups [19,20], and c) above $240 \mathrm{~K}$ (range C) there is a drastic increase of both QISFs. The transition observed at $240 \mathrm{~K}$ is referred to as "dynamical transition" corresponding to the onset of molecular motions on the picosecond-timescale. Qualitatively, the observed temperature dependence of molecular dynamics of PS II is similar to that of other biomolecular systems $[17,18,21,22]$, while specific transition temperatures and motional amplitudes differ. Later, QENS studies were reported for the bacterial RC of Rhodobacter sphaeroides and of two of its site-specific mutants [16]. The data reveal a dynamical transition at about $230 \mathrm{~K}$, i.e. at a rather similar temperature as observed for PS II from spinach. Remarkably, the two non-functional mutants of the bacterial RC were found to be more flexible than the wild type above the dynamical transition, see below.

As suggested by the data shown in Fig. 3, the dynamical transition in PS II membrane fragments is hydration dependent and found to be absent in dry 


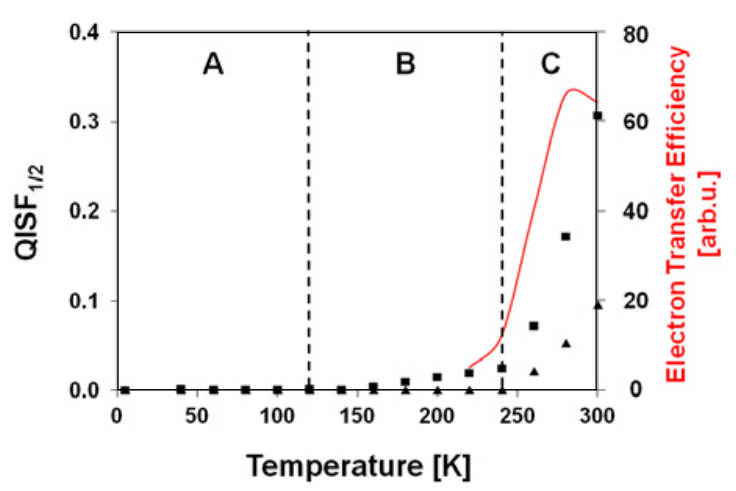

Figure 4. Temperature dependence of the slow ( $\mathrm{QISF}_{1}$, squares) and fast $\left(\mathrm{QISF}_{2}\right.$, triangles) quasielastic components obtained from QENS data of PS II membrane fragments hydrated at $90 \%$ r.h. according to [10]. The three temperature ranges A, B, and $\mathrm{C}$ are discussed in the text. The electron transfer efficiency according to [6] is shown by a full red line. This figure is reprinted from [10] with permission. Copyright (2007) American Chemical Society.

samples [10]. This finding was later verified by ENS experiments with a time resolution of about $100 \mathrm{ps}$ on samples hydrated at $44 \%$ and $57 \%$ r.h., respectively [13]. The data confirm that a dynamical transition is present in PS II membrane fragments hydrated at 57\% r.h., but absent at $44 \%$. These findings corroborate the above conclusion that dynamics in PS II membrane fragments sets on above $44 \%$ r.h.. Furthermore, ENS was used to study the dynamics of PS II membrane fragments up to a temperature of $340 \mathrm{~K}$ [14]. The data reveal a further hydration-sensitive transition between above $310 \mathrm{~K}$ that was attributed to the detachment of the oxygen evolving complex.

\section{Dynamics-function correlation}

Figure 4 also shows the temperature dependence of the $\mathrm{Q}_{\mathrm{A}} \rightarrow \mathrm{Q}_{\mathrm{B}}$ electron transfer efficiency in the PS II RC gathered from fluorescence quantum yield experiments [6]. A comparison with the QENS data of PS II membrane fragments suggests that there is a close correlation between the thermal activation of molecular dynamics and the electron transfer efficiency from $\mathrm{Q}_{\mathrm{A}}$ to $\mathrm{Q}_{\mathrm{B}}[10,13]$. At the same time, the suppression of molecular dynamics upon dehydration $[10,11,13]$ seems to be responsible for the inhibition of $\mathrm{Q}_{\mathrm{A}}$ to $\mathrm{Q}_{\mathrm{B}}$ electron transfer below $45 \%$ r.h. [7].

The detailed mechanism of the conformational triggering of $\mathrm{Q}_{\mathrm{A}} \rightarrow \mathrm{Q}_{\mathrm{B}}$ electron transfer in the PS II RC is not yet clarified, but it can be speculated that changes of the $\mathrm{Q}_{B}$ structure and/or rearrangements of hydrogen bonds in the vicinity of the quinone binding site are required (see e.g. $[1,2,10,23])$. It is interesting to note that a similar correlation between molecular dynamics and $\mathrm{Q}_{\mathrm{A}} \rightarrow \mathrm{Q}_{\mathrm{B}}$ electron transfer was observed in the bacterial RC [24]. Indeed, in the case of the RC of Rhodobacter sphaeroides a reorientation of the $\mathrm{Q}_{\mathrm{B}}$ headgroup upon electron uptake was reported based on X-ray crystallography as shown in Fig. 5 [25]. In addition, however, the conformational changes required for $\mathrm{Q}_{\mathrm{B}}$ reduction by $\mathrm{Q}_{\mathrm{A}}$ appear to go beyond a simple gating mechanism, but rather also

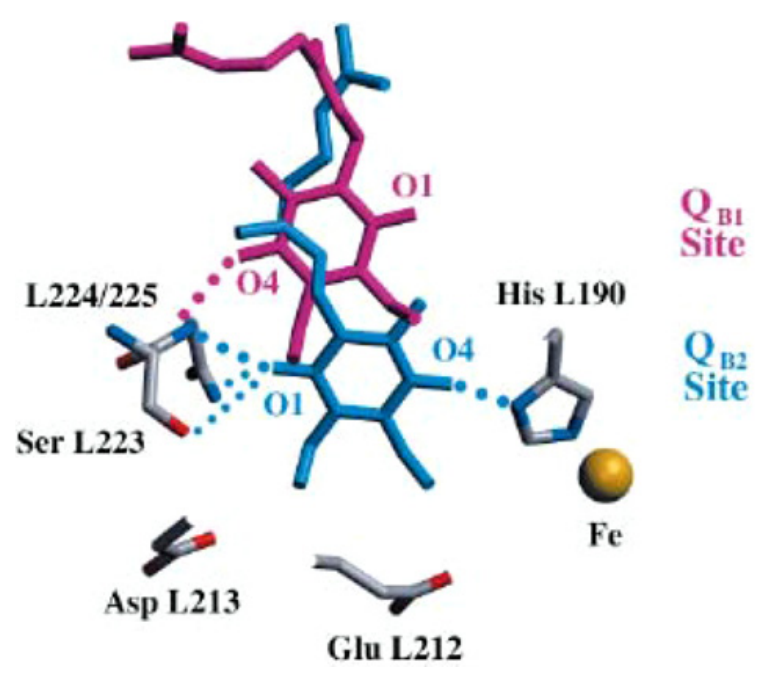

Figure 5. Conformational changes in the vicinity of the $\mathrm{Q}_{B}$ binding site upon $\mathrm{Q}_{\mathrm{A}} \rightarrow \mathrm{Q}_{\mathrm{B}}$ electron transfer according to Stowell et al. [25], see text. This figure is reprinted from [25] with permission.

comprise the rearrangement of hydrogen bonds, e.g. the reorientation of the Ser223 residue in the vicinity of the $\mathrm{Q}_{\mathrm{B}}$ binding site $[26,27]$. The latter conformational changes may likely be possible only upon activation of molecular dynamics as a "lubricant" $[10,13]$. In addition, the finding that the native bacterial $\mathrm{RC}$ is more rigid than two nonfunctional mutants [16] indicates that a specific flexibility is necessary for proper functioning.

In this regard, it is interesting to note that a functional role of molecular dynamics can also be inferred for certain redox reactions of the water oxidation in the PS II RC (see [1] and references therein) and for distinct electron transfer reactions in Photosystem I [28] based on the observed dependence on temperature. In summary, these findings underline that molecular dynamics plays a key functional role as demonstrated here for the case of electron transfer and plastoquinol formation in the PS II RC.

\section{Vibrational dynamics}

Information on vibrational dynamics of PS II membrane fragments was gathered from INS experiments [13] performed in a similar temperature range as covered by the QENS measurements discussed above. In antenna complexes, protein vibrations mediate excitation energy transfer towards the RC, so that determination of the vibrational density of states is a prerequisite for a proper understanding of these functional processes [29]. INS spectra were obtained for a PS II membrane fragment sample hydrated at $57 \%$ r.h. at temperatures of 20,150 , and $250 \mathrm{~K}$ in order to characterize the lowfrequency vibrations. The Boson peak energies are shown in Fig. 6 (red points) and compared to the average atomic mean square displacements $\left\langle\mathrm{u}^{2}\right\rangle$ of PS II membrane fragments equilibrated at different hydration levels $[10,13]$. The INS spectra exhibit a distinct Boson peak at $\sim 7.5 \mathrm{meV}$ at $20 \mathrm{~K}$, which shifts to $2.5 \mathrm{meV}$ at $250 \mathrm{~K}$. This observation differs from the low-temperature 


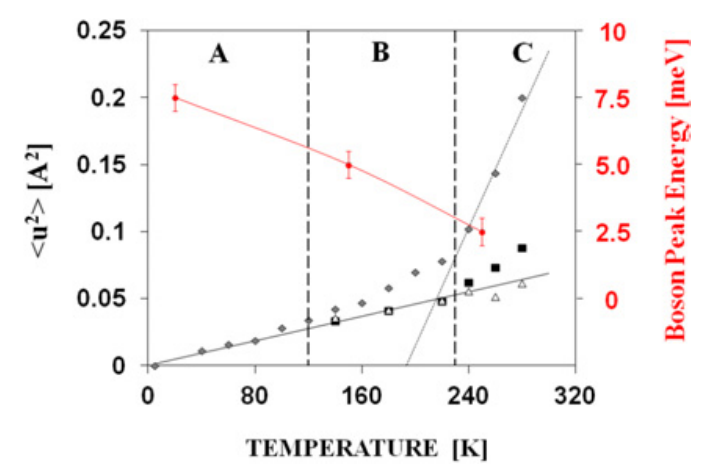

Figure 6. Comparison of Boson peak (red) derived from INS experiments at the NERA spectrometer and mean square displacements $<\mathrm{u}^{2}>$ of PS II membrane fragments obtained at different hydration levels and time resolutions. The $\left\langle\mathrm{u}^{2}>\right.$ - values correspond to hydration levels of 57\% r.h. (squares) and $44 \%$ r.h. (triangles) at a time resolution of $\sim 100 \mathrm{ps}$ at the IN13 spectrometer in this study (see Fig. 1) as well as for PS II membrane fragments hydrated at $90 \%$ r.h. (diamonds) at a time resolution of $\sim 20 \mathrm{ps}$. The temperature regions $\mathrm{A}, \mathrm{B}$, and $\mathrm{C}$ are the same as in Fig. 4. This figure is reprinted from [13] with permission.

behavior of the antenna complex LHC II, where the vibrational density of states was found to be widely similar up to $100 \mathrm{~K}[30,31]$. In contrast, the shift of the Boson peak in the case of PS II membrane fragments indicates a softening of the protein matrix along with the onset of molecular dynamics.

\section{Time-resolved QENS experiments}

As discussed above, the case of the $\mathrm{Q}_{\mathrm{A}} \rightarrow \mathrm{Q}_{\mathrm{B}}$ electron transfer in the PS II RC appears to be a striking example for a dynamics-function correlation. Functional activity and molecular dynamics can be simulataneously monitored in photosensitive biomolecular systems using the timeresolved laser-neutron pump-probe technique [32-35].

The principle of a time-resolved QENS experiment is illustrated in Fig. 7. The neutron probe pulse (n) probes the sample in a functional state initiated by the laser pulse, while neutron pulses $(n-1)$ and $(n+1)$ test the sample state before and after this instance, respectively. The neutron flight paths after interaction with the sample are depicted by dashed arrows. Figure 7 also shows the flash-induced fluorescence quantum yield transients of a spinach leaf as a red line and of PS II membrane fragments hydrated at $90 \%$ r.h. as a blue line. The decay of the red curve with a time constant of $\sim 160 \mu$ s is mainly determined by $\mathrm{Q}_{\mathrm{A}} \rightarrow \mathrm{Q}_{\mathrm{B}}$ electron transfer and thus sets the time scale of the time-resolved QENS experiment. A comparison of the two data sets shows that $\mathrm{Q}_{\mathrm{A}} \rightarrow \mathrm{Q}_{\mathrm{B}}$ electron transfer is not observed in PS II membrane fragments due to a limited pool of $\mathrm{Q}_{\mathrm{B}}$ molecules.

Following the first successful application of timeresolved QENS in the case of purple membrane [32], the novel technique was also applied to PS II membrane fragments [12]. The flash-induced QENS spectrum of PS II membrane fragments with impaired $\mathrm{Q}_{\mathrm{A}} \rightarrow \mathrm{Q}_{\mathrm{B}}$ electron transfer shown as a red line in Fig. 8 was measured

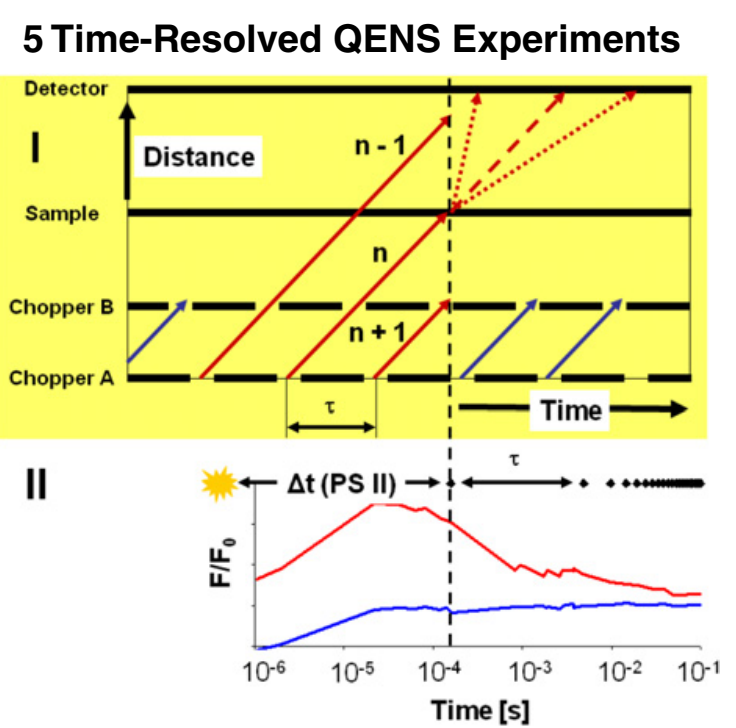

Figure 7. Schematic representation of time-resolved (laserneutron) pump-probe experiments: the synchronization of a pulsed QENS measurement (panel I) with laser activation of protein function (panel II). Note that for better illustration the timescale in panel I is linear, but logarithmic in panel II. The scales coincide when the neutron probe pulse $\mathrm{n}$ arrives at the sample (see vertical dashed line). Panel I: Scheme of pulsed QENS in a time-of-flight diagram. Monochromatic neutron pulses (see full arrows) are selected by a set of rotating choppers $\mathrm{A}$ and $\mathrm{B}$ with a repetition time $\tau$. The neutron probe pulse (n) arrives at the sample during the functional state prepared by the laser pulse, while neutron pulses $(n-1)$ and $(n+1)$ arrive before and after the laser-induced state of the sample, respectively. The dashed arrows indicate the neutron flight paths after interaction with the sample. Panel II: Flash-induced fluorescence quantum yield of an intact spinach leaf (red line) and of PSII membrane fragments with inhibited $\mathrm{Q}_{\mathrm{A}} \rightarrow \mathrm{Q}_{\mathrm{B}}$ electron transfer hydrated at $90 \%$ r.h. (blue line). The actinic laser flashes at $\mathrm{t}=0$ had a wavelength of $532 \mathrm{~nm}$ and a pulse energy of $8 \mathrm{~mJ} / \mathrm{cm}^{2}$. The decay of this curve is mainly determined by $\mathrm{Q}_{\mathrm{A}} \rightarrow \mathrm{Q}_{\mathrm{B}}$ electron transfer and, thus, defines the timescale of the PS II laser-QENS experiment. Black diamonds indicate the arrival times of the neutron pulses with a repetition time $\tau$ at the sample position. The value $\Delta \mathrm{t}$ denotes the time delay between laser flash and the neutron pulse. This figure is reprinted from [35] with permission.

selectively at $\sim 160 \mu$ s after the laser flash. A QENS spectrum obtained in the dark shown as black line in Fig. 8 reveals a statistically significant difference from the flash-induced QENS spectrum. A comparison of the three fractional quasielastic intensities (QISFs) obtained by fits of both spectra show that upon laser excitation the intensity of a slow 12 ps component increases at the expense of the fast $0.4 \mathrm{ps}$ term. This can be interpreted as a flash-induced transient suppression of molecular dynamics in PS II membrane fragments with inhibited $\mathrm{Q}_{\mathrm{A}} \rightarrow \mathrm{Q}_{\mathrm{B}}$ electron transfer [12]. Interestingly, this is the opposite phenomenon than the transient softening observed in the M-intermediate of bacteriorhodopsin [32].

The suppression of molecular dynamics found in PS II membrane fragments may possibly just reflect a change of the potential energy landscape upon laser illumination 

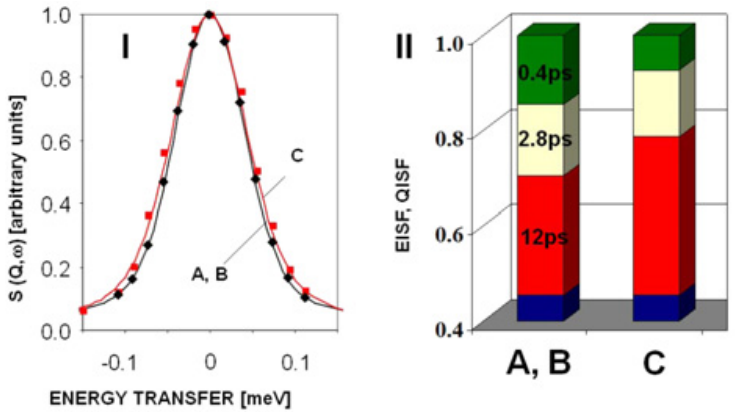

Figure 8. Left: normalized QENS spectra of PS II membrane fragments with inhibited $\mathrm{Q}_{\mathrm{A}} \rightarrow \mathrm{Q}_{\mathrm{B}}$ electron transfer (hydrated at $90 \%$ r. h. $\mathrm{D}_{2} \mathrm{O}$ ) obtained without (black) and with laser flash excitation (red) using a time delay of $\sim 160 \mu \mathrm{s}$. The spectra were measured at the time-of-flight spectrometer NEAT with an incident neutron wavelength of $5.1>\mathrm{A}$ and an elastic resolution of $93 \mu \mathrm{eV}$ (about $20 \mathrm{ps}$ ) at $295 \mathrm{~K}$. QENS data are averaged over all scattering angles so that the elastic $\mathrm{Q}=1.51>\AA^{-1}$. The laser-pumped experiments employed light flashes with a wavelength of $532 \mathrm{~nm}$ and a pulse energy of $8 \mathrm{~mJ} / \mathrm{cm}^{2}$ at $\mathrm{t}=0$. The black and red lines are fits with three Lorentzian components. The experimental uncertainty is similar to the size of the symbols. Right: QISFs (red, yellow, green) and EISF (blue) derived from the fits shown in the left frame. The QISFs are given with their characteristic times of $0.4,2.8$, and $12 \mathrm{ps}$. The letters A, B, and $\mathrm{C}$ correspond to the fit functions of A) dark measurement and C) laser-excited experiment at $\sim 160 \mu \mathrm{s}$ after the laser flash. The figure is reprinted from [35] with permission.

and upon formation of $\mathrm{Q}_{\mathrm{A}}$. However, the flash-induced fluorescence quantum yield transients of Fig. 7 indicate the formation of carotenoid triplet states ${ }^{3} \mathrm{Car}$. Therefore, the suppression of molecular dynamics may also correspond to aggregation-like conformational changes induced by ${ }^{3}$ Car formation. Aggregation may be linked to proposed mechanisms of photoprotection in the PS II antenna, which are capable of dissipation of excess light energy [36-38]. Although further studies are required, the observation of reduced molecular dynamics shows that the PS II protein matrix reveals a remarkable dynamical flexibility capable of accomodating different functional properties. As such, this result illustrates the potential of the time-resolved QENS technique to address questions on correlations between structure, dynamics and function even in complex systems like PS II.

\section{Conclusions}

In summary, the temperature- and hydration-dependence of molecular dynamics in PS II membrane fragments shows properties quite typical for biomolecules and biomembranes in general. Below $120 \mathrm{~K}$, mainly harmonic vibrational dynamics is observed, while (conformational) molecular dynamics is frozen. There is a partial activation of molecular dynamics at $120 \mathrm{~K}$ as well as a hydrationdependent "dynamical transition" at $240 \mathrm{~K}$. However, it is an intriguing phenomenon that the activation of molecular dynamics at $240 \mathrm{~K}$ appears to be a precondition for important functional properties illustrated by a rather similar temperature dependence of the $\mathrm{Q}_{\mathrm{A}} \rightarrow \mathrm{Q}_{\mathrm{B}}$ electron transfer efficiency in the PS II RC. In this regard, it is necessary to add that a functional role of molecular dynamics can also be anticipated for distinct reactions sequences of the water oxidation in the PS II RC and for specific electron transfer reactions in Photosystem I based on the observed dependence on temperature. In summary, these findings underline that molecular dynamics plays a key functional role, while electron transfer in Photosystem II can be viewed as an example for a dynamics-function correlation.

This paper is dedicated to the memory of Prof. Dr. Gernot Renger and summarizes the results of more than ten years of fruitful and pleasant scientific cooperation. Furthermore, the author thanks T. Hau $\beta$, R.E. Lechner, N.A. Dencher and J. Peters for helpful discussions. J.P. is grateful for financial support by the European Social Fund's Doctoral Studies and Internationalisation Programme DoRa, for funding by the Estonian Research Council (Grants ETF 9453, IUT 2-28 and SLOKT 12026 T) as well as to HZB Berlin, ILL Grenoble and JINR Dubna for the beam time.

\section{References}

[1] G. Renger, Biochim. Biophys. Acta 1817, 1164 (2012)

[2] F. Müh, C. Glöckner, J. Hellmich, A. Zouni, Biochim. Biophys. Acta 1817, 44 (2012)

[3] A. Zouni, H.-T. Witt, J. Kern, P. Fromme, N. Krauß, W. Saenger, P. Orth, Nature 409, 739 (2001)

[4] J. Bernarding, H.-J. Eckert, H.-J. Eichler, A. Napiwotzki, G. Renger Photochem. Photobiol. 59, 566 (1994)

[5] G. Renger, Biochim Biophys. Acta 1655, 195 (2004)

[6] G. Renger, H. M. Gleiter, E. Haag, F. Reifarth, Z. Naturforsch. 48c, 234 (1993)

[7] O. Kaminskaya, G. Renger, V. A. Shuvalow Biochemistry 42, 8119 (2003)

[8] F. Gabel, D. Bicout, U. Lehnert, M. Tehei, M. Weik, G. Zaccai Q. Rev. Biophys. 35, 327 (2002)

[9] J. Pieper, G. Renger, Photosynth. Res. 102, 281 (2009)

[10] J. Pieper, T. Hauß, A. Buchsteiner, K. Baczynski, K. Adamiak, R.E. Lechner, G. Renger, Biochemistry 46, 11398 (2007)

[11] J. Pieper, T. Hauß, A. Buchsteiner, G. Renger, Eur. Biophys. J. 37, 657 (2008)

[12] J. Pieper, G. Renger, Biochemistry 48(26), 6111 (2009)

[13] J. Pieper, M. Trapp, A. Skomorokhov, I. Natkaniec, J. Peters, G. Renger, Biochim. Biophys. Acta 1817, 2013 (2012)

[14] G. Nagy, J. Pieper, S. B. Krumova, L. Kovacs, M. Trapp, G. Garab, J. Peters, Photosynth. Res. 111, 113 (2012)

[15] A. Gall, J. Seguin, B. Robert, M.-C. Bellissent Funel, J. Phys. Chem. B 106, 6303 (2004)

[16] S. Sacquin-Mora, P. Sebban, V. Derrien, B. Frick, R. Lavery C. Alba-Simionesco Biochemistry 46(51), 14960 (2007)

[17] M. Ferrand, A.J. Dianoux, W. Petry, G. Zaccai, Proc. Natl. Acad. Sci. U.S.A. 90, 9668 (1993) 
[18] J. Fitter, R.E. Lechner, N.A. Dencher, J. Phys. Chem. B 103, 8036 (1999)

[19] J. E. Curtis, M. Tarek, D. J. Tobias J. Am. Chem. Soc. 126, 15928 (2004)

[20] J. H. Roh, V. N. Novikov, R. B. Gregory, J. E. Curtis, Z. Chowdhuri, A. P. Sokolov Phys Rev Lett 95, 038101 (2005)

[21] W. Doster, S. Cusack, W. Petry, Nature 337, 754 (1989)

[22] W. Doster, Eur. Biophys. J. 37, 591 (2008)

[23] P. Kühn, J. Pieper, O. Kaminskaya, H.-J. Eckert, R. E. Lechner, V. Shuvalov, G. Renger, Photosyn. Res. 84, 317 (2005)

[24] A. Garbers, J. Kurreck, F. Reifarth, G. Renger, F. Parak, Biochemistry 37, 11399 (1998)

[25] M. H. B. Stowell, T. M. McPhilips, D. C. Rees, S. M. Soltis, E. Abresch, G. Feher, Science 276, 812 (1997)

[26] R. H. G. Baxter, N. Ponomarenko, V. Srajer, R. Pahl, K. Moffat, J. R. Norris, Proc. Natl. Acad. Sci. U.S.A. 101, 5982 (2004)

[27] A. Y. Mulkidjanian, M. A. Kozlova, D. A., Cherepanov, Biochem. Soc. Trans. 33, 845 (2005)

[28] E. Schlodder, K. Falkenberg, M. Gergeleit, K. Brettel, Biochemistry 37, 9466 (1998)
[29] R. Jankowiak, M. Reppert, V. Zazubovich, J. Pieper, T. Reinot, Chem. Rev. 111, 4546 (2011)

[30] J. Pieper, K-D. Irrgang, G. Renger, R.E. Lechner, J. Phys. Chem. B 108, 10556 (2004)

[31] J. Pieper, M. Rätsep, K.-D. Irrgang, A. Freiberg, J. Phys. Chem. B 113, 10870 (2009)

[32] J. Pieper, A. Buchsteiner, N.A. Dencher, R.E. Lechner, T. Hauß, Phys. Rev. Lett. 100, 228103 (2008)

[33] S. Combet, J. Pieper, F. Coneggo, J.-P. Ambroise, M.C. Bellissent-Funel, J.-M. Zanotti, Eur. Biophys. J. 37, 693 (2008)

[34] A. Furrer, A. Stoeckli, Phys. Rev. E 81 (1), 011901 (2010)

[35] J. Pieper, Biochim. Biophys. Acta 1804 (1), 83 (2010)

[36] J. Pieper, K.-D. Irrgang, M. Rätsep, R. Jankowiak, T. Schrötter, G. J. Small, J. Voigt, G. Renger, J. Phys. Chem. B 103, 2422 (1999)

[37] J. Standfuss, M. Lamborghini, W. Kühlbrandt, A. C. T. van Scheltinga, EMBO J. 24, 919 (2005)

[38] A: V. Ruban, R. Berera, C. Ilioaia, I. H. M. van Stokkum, J. T. M. Kennis, A. A. Pascal, H. van Amerongen, B. Robert, P. Horton, R. van Grondelle, R., Nature 450, 575 (2007) 